\title{
A Novel Conceptual Risk Management Model Based on the Future's Uncertainties
}

\author{
Reza Hafezi ${ }^{1,2}$, Amir Naser Akhavan ${ }^{3,2}$ \\ ${ }^{1}$ Amirkabir University of Technology (Tehran Polytechnic), Technology Foresight Group, \\ Department of Management, Science and Technology, \\ P.O. Box 15875-4413, Tehran, Iran, \\ Email:r.hafezi@aut.ac.ir
}

${ }^{3}$ Amirkabir University of Technology (Tehran Polytechnic), Department of Petroleum Engineering, P.O. Box 15875-4413, Tehran, Iran, Email:akhavan@aut.ac.ir

${ }^{2}$ Futures Studies Research Institute

\begin{abstract}
Controlling and managing risk is always a critical point for the managers, investors and even consultants. Having clear and better percepts about future can reduce the risk of fail. In addition it is important for a risk analyzer to consider the stakeholders views and senses about the risk. This research aimed at framing a comprehensive risk management model which builds up on stakeholder requirements and forecasting techniques. This conceptual model attempts to use the stakeholder's points of view to write prefer and probable scenarios to neglect critical risks and increase the chance of success in longterm plannings. Using futures studies methods prepare decision makers for the possible upcoming events and donate them a powerful tool to handle the uncertainties cover the future.
\end{abstract}

Keywords: Risk Management, Futures Studies, Stakeholders analysis, Scenario Planning.

Jel classification: A100,A140

\section{Introduction}

Controlling and managing risk is always a critical point for the managers, investors and even consultants. Risk, generally, returns to a harmful event that may occur but with no determined certainty. Since the probability of risk (or even other uncertain threats) occurrence is covered by the uncertainty which exist in future's trends, it is important to have a vast and reliable insight about the future and upcoming events those can affect our business deeply. A detailed guide line that explains where to integerate risk management activities in to the project is still missing (Islam, Mouratidis, \& Weippl, 2014). When risk factors are unknown for manager or beyond of his/her control and project environment it is difficult to control the risk.

Many managers and investors ignore considering risk in its real meaning due to its unclear aspects. Studying risk needs a meaningful vision about future so the practice of risk management and futures studies are parallel in many ways.

Futures Studies refers to tools (methods, methodologies, techniques and even the way of thinking) which aimed at discovering the future's trends accurately. Futures Studies opens the window to a future view that helps us to develop our alternatives and donates us a better vision about the different aspects of future's environment. In this research we attempt to give the concepts of tying risk management and fore-sight methods to help decision makers considering risk in an understandable way.

Risk management is to control and reduce loses, make identifications, analysis and measuring of risk factors and then take effective actions (include: measuring, monitoring, controlling and warning) in order to eliminate or weaken the risk of accidents and reduce the economic loss and casualties (Xufeng, Qinggui, \& Yaodong, 2012).

In this research we attempt to consider human factor as the key element of the proposed model. Furthermore the scope of this research is to uncover more dimensions of risks the project can face with the potential ability of futures studies methods in future's uncertainties projection. Our focus is realizing risks that existing in developing technologies considering social impacts and stakeholders percepts

As Mao mentioned, this approach includes two meanings: First, all management activities are taken by human that is the main object and important resources. Second is all kind of management process needs to be promote and run by human (F, 2000). But in our vision we take human into account as the main beneficiary who his/ her goals draws the mainstream of futuring process 
and at the last decisions which made by manager will touch him/ her. So management process totally fails, if the decision making team, manager and performers neglect of stakeholders. We consider the problem of risk management as a macro argument and try to give a clear and smooth vision to managers tackling this critical issue.

Since risk analysis and management are complex and critical issues, many researchers are interested in publishing manuscripts contain new models to handle risks in a better way. Here a brief literature review of manuscripts that proposed risk models in different areas.

Xufeng et al. gave definition and function expression of risk based on the study of the connotation of the risk and the method of risk management in coal mine. They divided the risk management system into two dimensions containing technical and humanistic systems. Finally the technologyhumanism double loop control model and mathematical model of risk management was built (Xufeng et al., 2012).

Zhiyao et al. proposed a high-risk customer management model based on rough set theory to overcome the weakness of traditional risk management model in processing historical data efficiently. They proposed a method to reduce the irrelevant indicators before generating rules using rough set and combined risk management and rough set theory in a good way to process the historical data (Zhiyao, Wang Moyu, Ma Xinke, \& Xiaoliu, 2012).

Huang et al. presented an assessment model consisting of five dimensions (financial performance, logistical support, service level, learning and innovation, and risk control) that examines quantity and quality factors for equipment risk management in the petrochemical industry using 13 strategy subjects and 78 performancemeasurement indicators (Rong-Hwa Huang, Chang-Lin Yang, \& Kao, 2012). Huang et al. developed a risk management model and a distributed decision making (DDM) model for risk management of virtual enterprise (VE). The model has two levels, namely, the top model and the base model, which describe the decision processes of the owner and the partners of the VE, respectively (Min Huang, Fu-Qiang Lu, Wai-Ki Ching, \& Siu, 2011).

Marcelino-Sádaba et al. introduced a risk management method based on studying 72 of Spanish companies considering the factors are usually neglected by small businesses (Sara Marcelino-Sádaba, Amaya Pérez-Ezcurdia, Angel M. Echeverría Lazcano, \& Villanueva, 2014). Orian and Gheres proposed a risk management model to identify risks for travel agencies in Romanian by factor analysis. Identifies risks were categorized under organizational, environmental, competitive, economic, political, those of infrastructure, circumstance, business deficiencies and specific (local) risk (Maria Oroian \& Gheres, 2012). Fang and Marle presented a decision support system for modeling and managing of project risks and risk interactions (Chao Fang \& Marle, 2012).

$\mathrm{Xu}$ et al. introduced a tri-level programming model for the three-stage supply chain management based on the Conditional Value-at-Risk (CVaR) measure of risk management to maximize material supplier and the manufacturer profit while maximizing retailer $\mathrm{CVaR}$ of expected profit (Xinsheng Xu, Zhiqing Meng, \& Shen, 2013). Talay and Zheng modeled the risk control problem as a two players (Trader versus Market) zero-sum stochastic differential game problem (Denis Talay $\&$ Zheng, 2002). Yuntao et al. presented a framework of comprehensive risk management system for the defense science and technology enterprises, combining the features of the defense science and technology enterprises and the advanced risk management standards (Guo Yuntao, Li Suike, \& Sijun, 2011).

Islam et al. proposed a Goal-driven Software Development Risk Management Model (GSRM) and its explicit integration into the requirements engineering phase and an empirical investigation result of applying GSRM into a project (Islam et al., 2014). Bowers and Khorakian presented a theoretical framework which combines the generic innovation process with project risk management. The proposed framework was used to analyze the current attitudes to managing innovation risk in a series of companies (John Bowers \& Alireza Khorakian, 2014).

Rampini et al. studied the trade-off between financing and risk management in a dynamic model of commodity price risk management and show that risk management is limited and that more financially constrained firms hedge less or not at all using the case study of fuel price risk management by airlines (Adriano A. Rampini, Amir Sufi, \& Viswanathan, 2014). Shi et al. proposed a mixed approach to explore the management of delivery risk of a construction program: building a delivery risk structure for a construction program as the foundation of risk qualitative and quantitative analysis; analyze risk magnitude and assess the efficiency of delivery methods by using fuzzy logic theory and DEA (Shi, Zhou, Xiao, Chen, \& Zuo, 2014).

He presented a model of integrated risk management for Build-Operate-Transfer (BOT) projects In order to improve management level and risk management ability for BOT projects $(\mathrm{He}$, 
2012). Bharathi et al. proposed a model to predict the impact of risk that will lead to the failure of Enterprise Resource Planning (ERP) implementation to bringing out a risk assessment model by selecting certain critical pitfalls related to the implementation phase of ERP (S. Vijayakumar Bharathi, R. Raman, \& Dhanya Pramod, 2014).

Hwang et al. investigated Risk Management in small projects in Singapore in terms of status, barriers and impact of RM on project performance. A questionnaire survey was conducted and data were collected from 668 projects submitted by 34 companies. Finally analysis results indicated a relatively low level of Risk Management implementation in small projects, and that "lack of time", "lack of budget", "low profit margin", and "not economical" were prominent barriers. Also, the results reported the positive correlation between RM implementation and improvement in quality, cost and schedule performance of small projects, respectively (Bon-Gang Hwang, Xianbo Zhao, \& Toh, 2014).

Woodeard et al. designed flood risk strategies to allow for flexible adaptive measures by investigating the concepts of real options and multiobjective optimization to evaluate potential flood risk management opportunities. Their research describes a decision support methodology that has the capability to assess the most appropriate set of interventions to make in a flood system and the opportune time to make these interventions, given the future uncertainties (Michelle Woodward, Zoran Kapelan, \& Gouldby, 2014).

Grace et al. investigated which aspects of enterprise risk management add value and showed that the use of economic capital models and dedicated risk managers improve operating performance (Martin F. Grace, J. Tyler Leverty, and, \& Shimpi, 2014). Lorca and Prina developed a methodology includes modeling the multivariate stochastic evolution of locational electricity prices, the construction of a scenario tree that represents this evolution, and the formulation and solution of a stochastic optimization model in order to presenting a medium term power portfolio optimization model for a power producer in a competitive electricity market, considering locational electricity prices and risk management (Álvaro Lorca \& José Prina, 2014).

$\mathrm{Mu}$ et al. developed a risk management capability (RMC) assessment model for subway project contractors and to assess the current overall RMC of subway project contractors in mainland China (Mu, Cheng, Chohr, \& Peng, 2014). Fito and Guitart presented a risk management approach led by business-level objectives (BLOs) of Cloud organizations in order to assist in business-driven selfmanaged Cloud providers, by facing uncertainties always present in their internal decision-making processes (Fitó \& Guitart, 2014). Feng et al. proposed a security risk analysis model (SRAM) to identify the causal relationships among risk factors and analyze the complexity and uncertainty of vulnerability propagation ((Feng, Wang, \& $\mathrm{Li}$, 2014).

Some models even built up on a multi-agent frame works. Multi-agent systems are a combination of intelligent elements, called agents that interact each other in order to achieve a series of goals. Qing et al. used employing such theories as immunology, multi-agent model construction and simulation, and emergency management system engineering, etc. to present a multi-agent system of risk identifier of emergency management (YANG Qing, MA Huimin, \& Yanling, 2011). And Bajo et al. proposed a multi-agent system especially created to detect risky situations and provide recommendations to the internal auditors of smallmedium enterprises (SMEs) (Javier Bajo, María L. Borrajo, Juan F. De Paz, Juan M. Corchado, \& Pellicer, 2012).

\section{The Proposed Model}

In this section we discuss our proposed model. At the first see a glance of the whole model. 


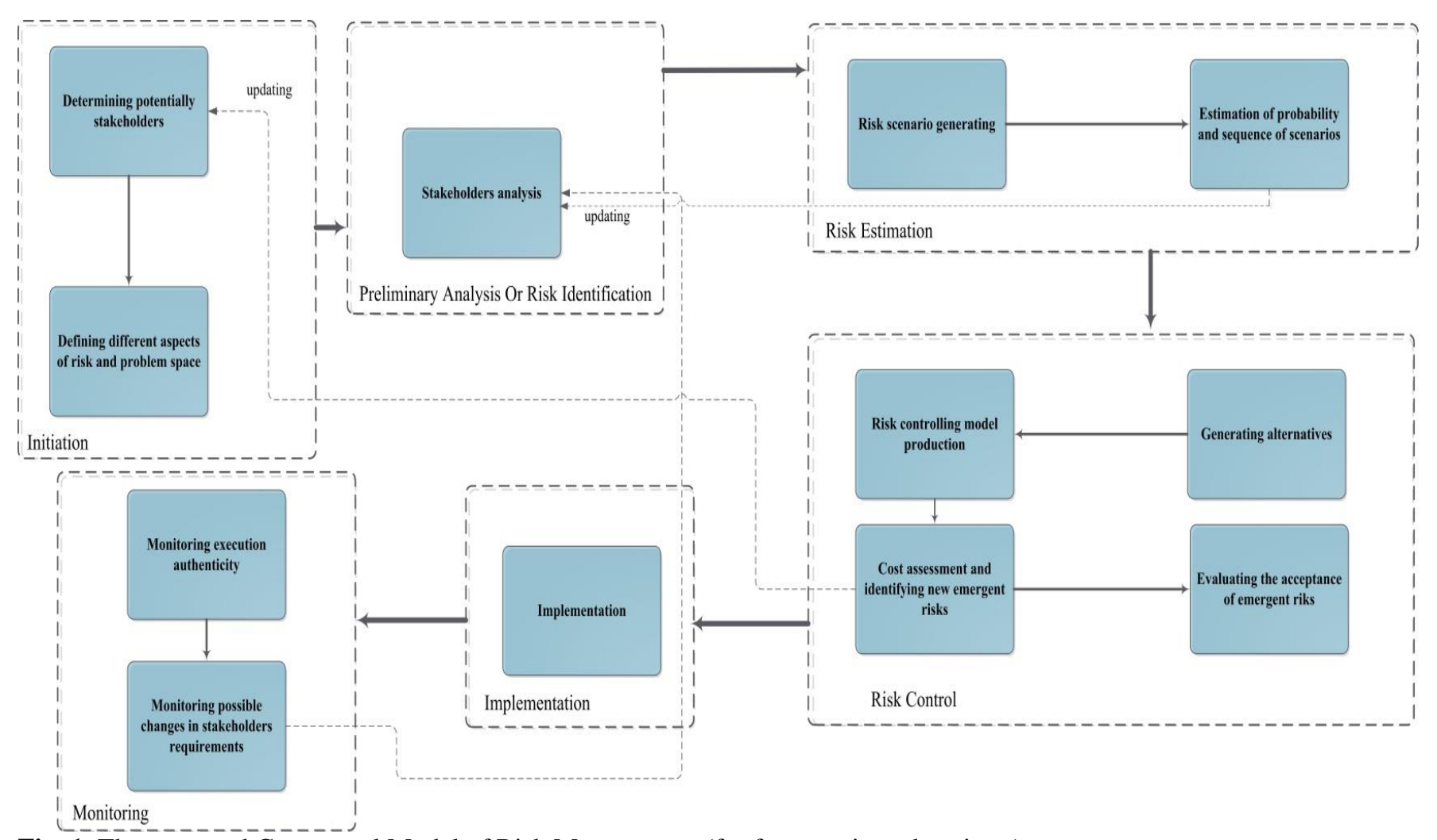

Fig. 1. The proposed Conceptual Model of Risk Management (for future-oriented projects)

\subsection{Initiation phase}

This phase containing two main processes aimed at determining the space of problem and actors. The first is finding potentially stakeholders who the benefits or losses would affect him/her or $\mathrm{s} / \mathrm{he}$ is the performer of decisions and anyone else who can affect our business or affected by our decisions. The second one is defining the different aspects of risk based on pre-determined stakeholders to clarify the problem space. It can be done using expert panel method that gets stakeholders and some selected experts together to communicate and discuss around the main issue. It helps decision makers, analyzers, futurist and ... to sawy how actors understand risk and makes a useful convergence among them.

\subsection{Preliminary Analysis or Risk Identification phase}

This phase gets the results and information rose from initiation phase and seeks to analyze stakeholders in order to figure it out their: requirements, disturbing and concerning issues, how they understanding risk, level of interest and knowledge about the problem/ subject, incorrect understandings and knowledge gaps and finding and creating reliable databases of information.

Interviewing and expert panels are useful methods to analyze stakeholders.

\subsection{Risk Estimation}

This phase is started by generating risk scenarios to capture the future of our current position under possible changes of uncertain factors and variables. A scenario is the splicer of future and today with a story of plausible cause and effect links while illustrating key decisions, events, and consequences throughout the narrative. In summery scenarios developed to: provide a list of unknowns that shall to be known, before making decisions, understanding the importance and effectiveness of uncertainties, determining what is possible and what is not, make the future more clear and real for decision makers to force new thinking and decisions and identifying what strategies might work across a range of possible scenarios. In simple words scenario prepares us to face future risks and uncovers new possible opportunities.

There are many ways to generate and develop scenarios. Here a two step process is illustrated:

\section{Step1.Prepration}

This step seeks to define the scenario space by giving a clear statement of domain and detecting a list of key driving forces that seem to shape future of interested domain. 


\section{Step2.Development}

This step contains of four sub-steps: defining the key measures, defining events, projection of key measures and preparing descriptions.

The key measures need to be selected carefully. A measure can be defined as key measure if it potentially has a great impact on outcomes. An irrelevant key measure explains as the factor which can change greatly over wide spectrum of values but these changes affect the scenario too little.

Events that appear in different scenarios can shape them in some ways: They can impact on chains of casualty that connect present to future, they can change key measures and in some cases they are able to make certain policies more or less likely to work. The probabilities of events differ in scenarios and it depends on the problem space.

To project key measures, trend impact analysis is a good technique. It started projection of collected historical data of measures using time series methods. Then to generate new forecasts and range of uncertainties Monte Carlo methods are used to extrapolate events probability.

Now the quantitative products (forecast results, probabilistic of events and casualty chains) are prepared so it is the time to tell cohesive narratives about the future.

This is not a really developed scenario. In addition to these two steps a complete scenario needs a third step to report and utilize (containing of three sub-steps: documentation, contrasting the implementations and the alternative worlds, testing policies) which is useless for this research, because we developed our own releasing and testing procedure based on stakeholders and actors that are the key point in management.

After the scenarios developed it is the time to estimate the probability of scenarios and their sequences. To achieve this goal we can implement environmental scanning. Environmental scanning is a process of scanning input generator elements, such as newsletters, journals, specific websites and etc. to identify and track the changes. Then it is important and necessary to update the stakeholder analysis step with new findings of this stage.

\subsection{Risk Estimation phase}

After scenarios generated it is the time to estimate risks and produce a good controlling model. This phase contains of four main parts. First you have you have to generate alternatives based on built scenarios and stakeholder's coordination. This can be done using Expert panels and Delphi methods. It is important to evaluate stakeholders feeling about risk and their level of risk taking.

Outcomes send to a node to produce and develop a risk controlling model. The proposed model has to be check with stakeholders and get the agreement because the model is developed to answers their requirements.

After the model is produced and got stakeholders agreement the cost of model has to be assessed and new emergent risks must be identified. Agent-based simulation and environmental scanning are two appropriate methods for handling this job. In the processing of previous stage new stakeholders are identified and identifying stakeholders phase must be upgrade. When new risks are identified they have to be checked to deliver key actors acceptance via expert panels.

\subsection{Implementing phase}

This phase contains implementing of proposed model. It is important to engaging stakeholders. In this model we seek to build a good model which has stakeholders in its neighbor. If the best model with optimized variables has been proposed by risk management team with no execution guarantee from stakeholders is nothing but a good model with this guarantee will work.

\subsection{Monitoring phase}

Monitoring phase has two sub-phases containing: monitoring the implementation authenticity and monitoring the possible changes in stakeholder's requirements over the time.

\section{Conclusions}

Having clear and better percepts about future can reduce the risk of fail. One of the conditions for meeting success in today's challenging world is the ability of designing plans and strategies to handle probable futures through improving information flows and decreasing the risk of failures. During past decades risk management has become a substantial topic both in industries and academic views. Unknown and sophisticate environment where decisions are made make managers to focus on the probable risks and social views specialy in developing new technologies and policies.

In this research we tried to combine advantages of futures studies in eliminating dusts that cover the image of future in decision makers mind and risk management to shape the long-term decisions. In order to achieve this goal a conceptual framework for risk management developed to 
consider futures studies methods and stakeholders point of view. The proposed model contains scenarios to discover the uncertainties of future and organize manager's, leader's, policy maker's and stakeholder's insights. Futures studies methods help organizations (of a vriety of types from firms to goverments) to avoid unexpected issues in probable futures.

\section{References (at least 25-30)}

Adriano A. Rampini, Amir Sufi, \& Viswanathan, S. (2014). Dynamic risk management. Journal of Financial Economics, 111(2), 271-296.

Álvaro Lorca, \& José Prina. (2014). Power portfolio optimization considering locational electricity prices and risk management. Electric Power Systems Research, 109, 80-89.

Bon-Gang Hwang, Xianbo Zhao, \& Toh, Li Ping. (2014). Risk management in small construction projects in Singapore: Status, barriers and impact. International Journal of Project Management, 32(1), 116-124.

Chao Fang , \& Marle, Franck. (2012). A simulationbased risk network model for decision support in project risk management. Decision Support Systems, 52, 635-644.

Denis Talay, \& Zheng, Ziyu. (2002). Worst case model risk management. Finance Stochast, 6, 517-537.

F, Mao H. (2000). Modern safety management theory and practice: Capital Economic and Trade University Press.

Feng, Nan, Wang, Harry Jiannan, \& Li, Minqiang. (2014). A security risk analysis model for information systems: Causal relationships of risk factors and vulnerability propagation analysis. Information Sciences, 256, 57-73.

Fitó, J. Oriol, \& Guitart, Jordi. (2014). Business-driven management of infrastructure-level risks in Cloud providers. Future Generation Computer Systems, 32, 4153.

Guo Yuntao, Li Suike, \& Sijun, Bai. (2011). Framework of Comprehensive Risk Management System for the Defense Science and Technology Enterprises. Paper presented at the 2011 International Conference on Business Management and Electronic Information (BMEI), Guangzhou.

He, Jun. (2012). Study for Integrated Risk Management of BOT Projects. Civil Engineering and Urban Planning 2012, 594-598.

Islam, Shareeful, Mouratidis, Haralambos, \& Weippl, Edgar R. (2014). An empirical study on the implementation and evaluation of a goal-driven software development risk management model. Information and Software Technology, 56(2), 117-133.

Javier Bajo, María L. Borrajo, Juan F. De Paz, Juan M. Corchado, \& Pellicer, María A. (2012). A multi-agent system for web-based risk management in small and medium business. Expert Systems with Applications, 39, 6921-6931.
John Bowers, \& Alireza Khorakian. (2014). Integrating risk management in the innovation project. European Journal of Innovation Management, 17(1), 25-40.

Maria Oroian, \& Gheres, Marinela. (2012). Developing a risk management model in travel agencies activity: An empirical analysis. Tourism Management, 33, 15981603.

Martin F. Grace, J. Tyler Leverty, and, Richard D. Phillips, \& Shimpi, Prakash. (2014). The Value of Investing in Enterprise Risk Management. The Journal of Risk and Insurance. doi: 10.1111/jori.12022

Michelle Woodward, Zoran Kapelan, \& Gouldby, Ben. (2014). Adaptive Flood Risk Management Under Climate Change Uncertainty Using Real Options and Optimization. Risk Analysis, 34(1), 75-92.

Min Huang, Fu-Qiang Lu, Wai-Ki Ching, \& Siu, Tak Kuen. (2011). A distributed decision making model for risk management of virtual enterprise. Expert Systems with Applications, 38, 13208-13215.

$\mathrm{Mu}$, Shiyu, Cheng, Hu, Chohr, Mohamed, \& Peng, Wei. (2014). Assessing risk management capability of contractors in subway projects in mainland China. International Journal of Project Management, 32(3), 452460.

Rong-Hwa Huang, Chang-Lin Yang , \& Kao, ChungSzu. (2012). Assessment model for equipment risk management: Petrochemical industry cases. Safety Science, 50, 1056-1066.

S. Vijayakumar Bharathi, R. Raman, \& Dhanya Pramod. (2014). A FPN Based Risk Assessment Model for ERP Implementation in Small and Medium Enterprises. Middle-East Journal of Scientific Research, 19(6), 747-759.

Sara Marcelino-Sádaba, Amaya Pérez-Ezcurdia, Angel M. Echeverría Lazcano, \& Villanueva, Pedro. (2014). Project risk management methodology for small firms. International Journal of Project Management, 32.

Shi, Qian, Zhou, Yikun, Xiao, Chao, Chen, Rongyi, \& Zuo, Jian. (2014). Delivery risk analysis within the context of program management using fuzzy logic and DEA: A China case study. International Journal of Project Management, 32(2), 341-349.

Xinsheng Xu, Zhiqing Meng, \& Shen, Rui. (2013). A tri-level programming model based on Conditional Value-at-Risk for three-stage supply chain management. Computers \& Industrial Engineering, 66, 470475.

Xufeng, PANG, Qinggui, CAO, \& Yaodong, JIANG. (2012). Research on the Double Loop Control Model of Risk Management in Coal Mine. Paper presented at the First International Symposium on Mine Safety Science and Engineering.

YANG Qing, MA Huimin, \& Yanling, YU. (2011). Multi-Agent Risk Identifier Model of Emergency Management System Engineering Based on Immunology. Paper presented at the Information Engineering and Complexity Science - Part II.

Zhiyao, Li, Wang Moyu, Ma Xinke, \& Xiaoliu, Shen. (2012). High Risk Management Model For The Power Enterprise Based on Rough Set Theory. Paper presented at the The 2nd International Conference on Complexity Science \& Information Engineering. 